\title{
Review of: "SARS-CoV-2 infectivity by viral load, S gene variants and demographic factors and the utility of lateral flow devices to prevent transmission"
}

Sylvie Pillet ${ }^{1}$

1 Université Jean Monnet

Potential competing interests: The author(s) declared that no potential competing interests exist.

Well written and scientifically sound article. I have no comment to make, except to change the 2 shades of yellow/orange in figure 5. I did not have access to figure S1.

https://doi.org/10.1101/2021.03.31.21254687 